\title{
Spatiotemporal changes of NDVI and correlation with meteorological factors in northern china from 1985-2015
}

\author{
Shun Pan $^{1}$, Xiangwei Zhao ${ }^{1, *}$, and Yaojie Yue ${ }^{2}$ \\ ${ }^{1}$ Key Laboratory of Geomatics and Digital Technology of Shandong Province, Shandong University of Science and Technology, \\ Qingdao 266590, China \\ ${ }^{2}$ School of Geography, Beijing Normal University, Beijing 100875, China
}

\begin{abstract}
The NDVI in northern china is vulnerable and is greatly affected by meteorological factors. In this study, the correlation between changes of NDVI and the major meteorological factors (temperature, precipitation) in northern China in the past 30 years with Trend analysis and partial correlation analysis methods. The results show that: (1) In the recent 30 years, the annual average NDVI in northern China shows an upward trend of volatility, with an annual growth rate of 0.0015 , and the overall vegetation coverage increases. (2) In the context of global warming, the average annual temperature in the research area increased significantly, with an average annual growth rate of 0.0353 , while the average annual growth rate of precipitation was not significant, with an average annual growth rate of 0.1591. (3) NDVI has a higher correlation with air temperature in high altitude alpine and plateau areas, and a higher correlation with precipitation in grassland and desert grassland areas.
\end{abstract}

\section{Introduction}

Vegetation as a major component of terrestrial ecosystems, it not only plays a major role in regulating biodiversity, but also has an important contribution to earth's climate regulation and soil and water conservation. The dynamic change of vegetation cover is closely related to environmental factors such as climate, soil and water, so it is regarded as the "indicator" of environmental ecology [1]. It is widely believed that climate change can cause changes in vegetation cover and may eventually lead to increased frequency and severity of regional extreme events (desertification, salinization, dust storms, floods etc.)[2]. Therefore, exploring the intrinsic relationship between the temporal and spatial changes of vegetation cover and climate change can reveal the evolution process of regional environmental conditions and predict the future development trend.

Normalized difference vegetation index (NDVI) is an important indicator for measuring vegetation coverage, which plays an important role in the research of global and regional land cover, vegetation dynamic change and phenological change [3]. In recent years, scholars have made remarkable achievements in the research on NDVI and its influencing factors. Guan et al. analyzed the changes of NDVI in the Hexi Corridor and its surrounding areas [4]. The NDVI in the Qilian Mountains is closely related to precipitation, but negatively correlated with precipitation in oasis area. Zhao et al. analyzed the relationship between NDVI and climate in the grassland region of northern China from 2000 to 2010 [5]. The results show that precipitation is the strongest positive driving force, followed by average temperature. Muradyan et al. concluded that temperature is positively correlated with meadow vegetation NDVI and negatively correlated with xerophytic/steep vegetation [6]. In addition, NDVI has a certain relationship with terrain and altitude. Vegetation in temperate mountainous areas is strongly influenced by topography, wind speed and sunshine [7], but vegetation with altitudes between 3000 and 3400 is susceptible to human activities.

Most of the above studies are focused on the regional and some sensitive areas, while there is little research on arid and semi-arid climate regions in northern China. In this paper, the spatial and temporal variation trend of NDVI and its correlation with meteorological factors were analyzed in 13 provinces in northern China. It can provide a basis for the monitoring and protection of vegetation in the north, provide support for the construction of ecological environment and sustainable development.

\section{Study Area}

The study area is located at $73-136^{\circ} \mathrm{E}$ longitude and 31 $54^{\circ} \mathrm{N}$ latitude, with a total area of $4.995 \times 10^{4} \mathrm{~km}^{2}$, including 13 provinces (municipalities) of Beijing, Hebei, Shaanxi, Tianjin, Jilin, Shanxi, Inner Mongolia, Ningxia, Liaoning, Gansu, Heilongjiang, Qinghai and Sinkiang(Fig.1). The climate of the study area is dominated by arid semi-arid and semi-humid climates. The precipitation is concentrated in July and August in summer, and the annual precipitation is $400 \sim 800 \mathrm{~mm}$. Most of the areas are located in the northern agro-pastoral

"Corresponding author: zhaoxwchina@ sdust.edu.cn; tlzxw1696@163.com; 
ecotone, and the land cover is mainly grassland, forests and gobi desert, and the ecological environment is relatively fragile. In addition, large areas of desertified land are distributed in Sinkiang, Gansu, Qinghai and Inner
Mongolia, and the areas bordering with them have single vegetation types, such as shrubs and grasslands, which are vulnerable to invasion and develop into the desert [4].

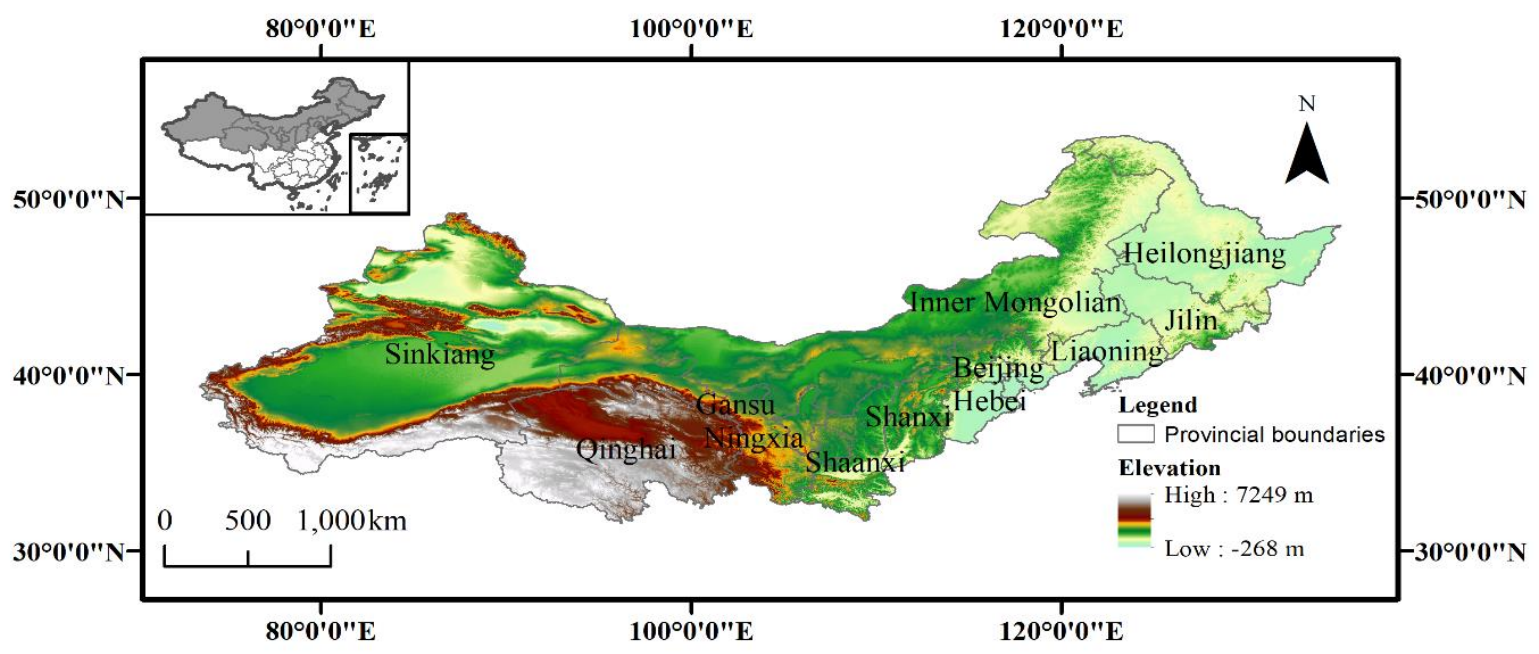

Fig.1. Location of the study area, North China.

\section{Data and methods}

\subsection{Data source}

The GIMMS NDVI3g data from the Advanced Very High Resolution Radiometer (AVHRR), provided by the Global Inventory Modeling and Mapping Study (GIMMS) group of NASA. The data set is a half-month synthetic data with a spatial resolution of $8 \mathrm{~km}$ and a time span of 1985-2015. The annual data obtained by applying a maximum value compositing (MVC) algorithm can minimize the influence of clouds and aerosols [8]. It has been subjected to a series of processing such as projection conversion, radiation calibration, geometric correction.

The meteorological data comes from the China Meteorological Science Data Sharing Service website (http://data.cma.cn/). Monthly standard meteorological data for 625 stations nationwide, including data on climate, wind speed, precipitation, humidity, and sunshine. Exceeding the annual average temperature and annual precipitation data from sites with more missing data. After projection, Kriging interpolation, and cutting, the data layer of the meteorological grid of the study area from 1985-2015 was obtained.

\subsection{Methods}

Unitary linearity regression method was used to estimate the linear trend of each pixel NDVI over time, which could reflect the changing trend of NDVI more reasonably and precisely [8]. The formula is expressed as follows:

$$
\theta_{\text {slope }}=\frac{n \times \sum_{i=1}^{n} i \times N D V I_{i}-\left(\sum_{i=1}^{n} i\right)\left(\sum_{i=1}^{n} N D V I_{i}\right)}{n \times \sum_{i=1}^{n} i^{2}-\left(\sum_{i=1}^{n} i\right)^{2}}
$$

where $i=1,2, \cdots 31, \mathrm{n}=31, N D V I_{i}$ represents the maximum annual NDVI value in the study area in year $i$. when $\theta_{\text {slope }}<0$ connotes the tendency of vegetation degradation; $\theta_{\text {slope }}>0$ indicates a restoration trend of vegetation; $\theta_{\text {slope }}=0$ means the vegetation NDVI changes are not obvious.

Climate change is one of the major factors affecting NDVI change in study area. Therefore, the correlation coefficient between annual maximum NDVI and annual average temperature and precipitation was calculated pixel by pixel to represent the degree of correlation between climate factors and NDVI changes. The formula is expressed as follows:

$$
R_{x y}=\frac{\sum_{i=1}^{n}\left[\left(x_{i}-\bar{x}\right)\left(y_{i}-\bar{y}\right)\right]}{\sqrt{\sum_{i=1}^{n}\left(x_{i}-\bar{x}\right)^{2} \sum_{i=1}^{n}\left(y_{i}-\bar{y}\right)^{2}}}
$$

Where $R_{x y}$ represents the correlation coefficient of the two variables $x$ and $y ; x_{i}$ and $y_{i}$ represent the values of the $i$ years of the two variables $x$ and $y$ respectively; $n$ represents the cumulative number of years, $n=31 ; \bar{x}$ and $\overline{\mathrm{y}}$ represent the average of the two variables in $n$ years.

Based on the simple linear correlation calculation, the partial correlation coefficient can be further derived. The formula is as follows:

$$
R_{x y, z}=\frac{R_{x y}-R_{x z} R_{y z}}{\sqrt{1-R_{x z}^{2}} \sqrt{1-R_{y z}^{2}}}
$$

Where $R_{x y, z}$ is the partial correlation coefficient between the variable $x$ and $y$ while keeping the variable $z$ constant.

\section{Result}

\subsection{Temporal and spatial variation trend of NDVI}

The NDVI of the study area was counted from 1985 to 2015, and the annual average NDVI dynamic curve was obtained (Fig.2). From the curve of annual average NDVI 
change, it can be seen that the NDVI of vegetation in northern China presents a slow increase trend as a whole, with an increase rate of 0.0015 . From the perspective of dynamic changes, from 1985 to 2008, the annual average annual NDVI showed a slow increase trend, and a sharp decrease in 1999 and 2000. After 2000, the slope of NDVI increased, the change volatility of NDVI increased after 2008 , but the overall increase was significant.

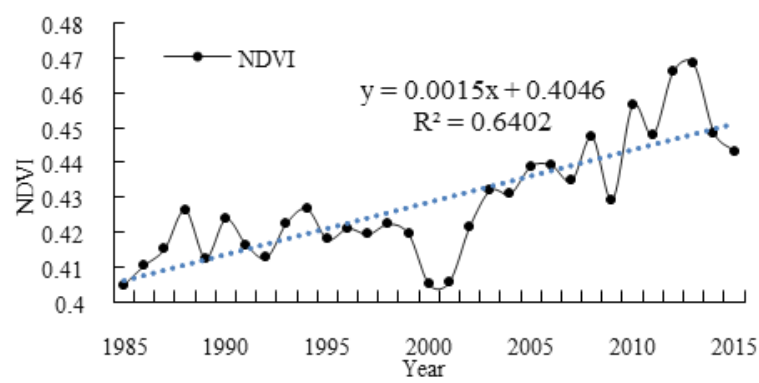

Fig. 2. Change trend of the average of annual NDVI.

The study area NDVI dynamic was calculated using equation (1), the spatial characteristics of vegetation NDVI change trend is shown in Fig.3. According to the value of $\theta_{\text {slope }}$, the variation trend of NDVI in the study area was divided into 5 grades, as shown in Table 1 . It can be seen from Figure. 3 and Table 1 that NDVI in northern China showed an increasing trend from 1985 to 2015, but there were obvious regional differences.

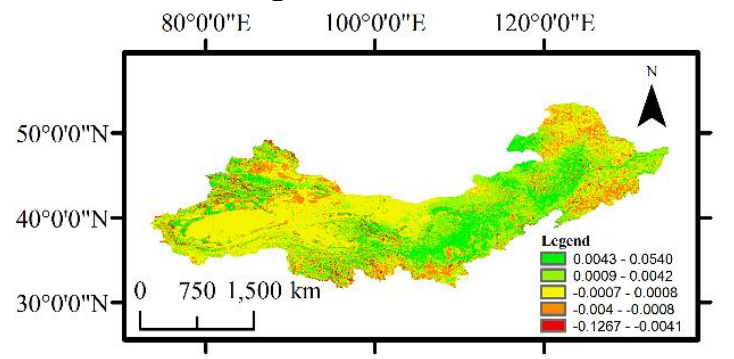

Fig. 3. The NDVI trends of study area during 1985-2015.

The areas with significant increase in vegetation NDVI are mainly distributed in Tianshan, Qilian Mountains, Loess Plateau, western Inner Mongolia and northwestern Heilongjiang; slightly increased areas are distributed in central Inner Mongolia and northeast plain. The area of NDVI with an increasing trend accounted for $46.9 \%$ of the total area of the study area. Vegetation NDVI showed a reduced area of $16.56 \%$, mainly distributed in the eastern part of Ningxia, southern Qinghai, the junction of Shaanxi and Ningxia, parts of Gansu and eastern part of Liaoning. The area with no obvious trend is $36.36 \%$, which is distributed in the Tarim Basin and the western part of the Inner Mongolia Plateau.

Table 1. Inter-annual variation trend of NDVI.

\begin{tabular}{|c|c|c|}
\hline Dynamic trend & $\boldsymbol{\theta}_{\text {slope }}(\boldsymbol{\theta})$ range & Area/\% \\
\hline Significant increase & $0.0043 \leq \theta \leq 0.0540$ & 17.13 \\
\hline Slightss increase & $0.0009 \leq \theta \leq 0.0042$ & 29.77 \\
\hline No obvious change & $-0.0007 \leq \theta \leq 0.0008$ & 36.36 \\
\hline Slight decrease & $-0.004 \leq \theta \leq-0.0008$ & 14.28 \\
\hline Significant decrease & $-0.1267 \leq \theta \leq-0.0041$ & 2.28 \\
\hline
\end{tabular}

\subsection{The relationship between NDVI and climate factors}

In the context of global warming, the temperature increase trend of the study area from 1985-2015 was significant (Fig.4). At the same time, precipitation also shows a slow increase trend (Fig.5), while precipitation increases slowly relative to the temperature growth rate. It can be seen from Figure. 4 that the annual average temperature in the research area rises at a rate of $0.0353^{\circ} \mathrm{C} \cdot \mathrm{a}^{-1}$, which is in good synchronism with the changing trend of NDVI, while the increase rate of annual precipitation in the research area is $0.1591 \mathrm{~mm} \cdot \mathrm{a}^{-1}$, which more obvious synchronism with the changing of NDVI value.

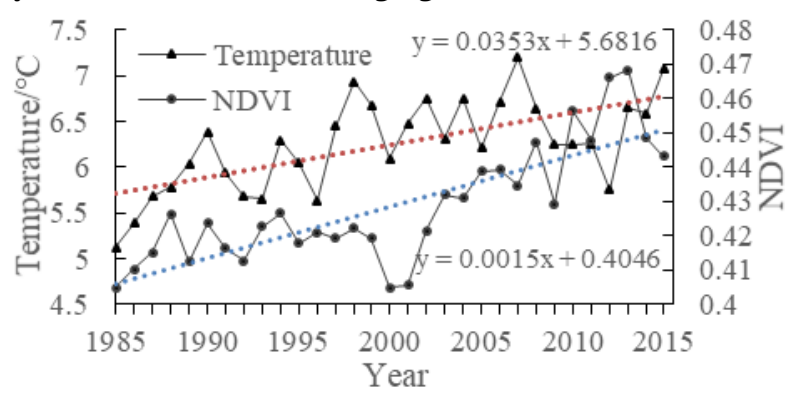

Fig. 4. Correlation between NDVI and Temperature

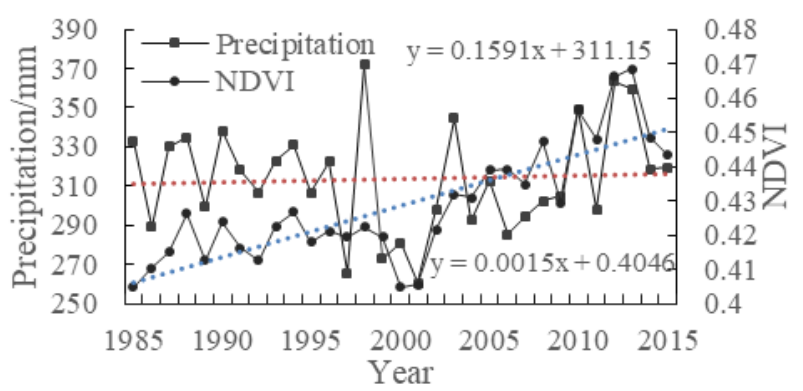

Fig. 5. Correlation between NDVI and Precipitation

Analysis of the spatial correlation between NDVI and temperature correlation coefficient (Fig.6a) and partial correlation coefficient (Fig.6b) shows that the two have similar spatial analysis characteristics. Temperature and NDVI extremely significant positive correlation (correlation $>0.5$ ) are distributed in the Qinghai-Tibet Plateau and the Qilian Mountains and the Taihang Mountains. The maximum correlation coefficient and partial correlation coefficient are 0.949 and 0.962 , respectively. The significant positive correlation $(0.2<$ correlation $<0.5)$ region is mainly distributed in the Tianshan Mountains, the south of the Junggar Basin in Xinjiang, the Kunlun Mountains, the lower reaches of the Tarim River, and parts of the Qinghai-Tibet Plateau. The average altitude in these areas is more than 3,000 meters, and the annual temperature is low, which limits the growth of vegetation. Other areas of significant positive correlation include Central Inner Mongolia and northern Heilongjiang. The areas of no obvious correlation $(-0.2<$ correlation $<0.2$ ) are mainly distributed in the Northeast Plain and the Tarim Basin. The land cover type in the Northeast Plain is cultivated land, and the growth of cropsis greatly disturbed by human factors, thus showing no significant correlation with temperature. 


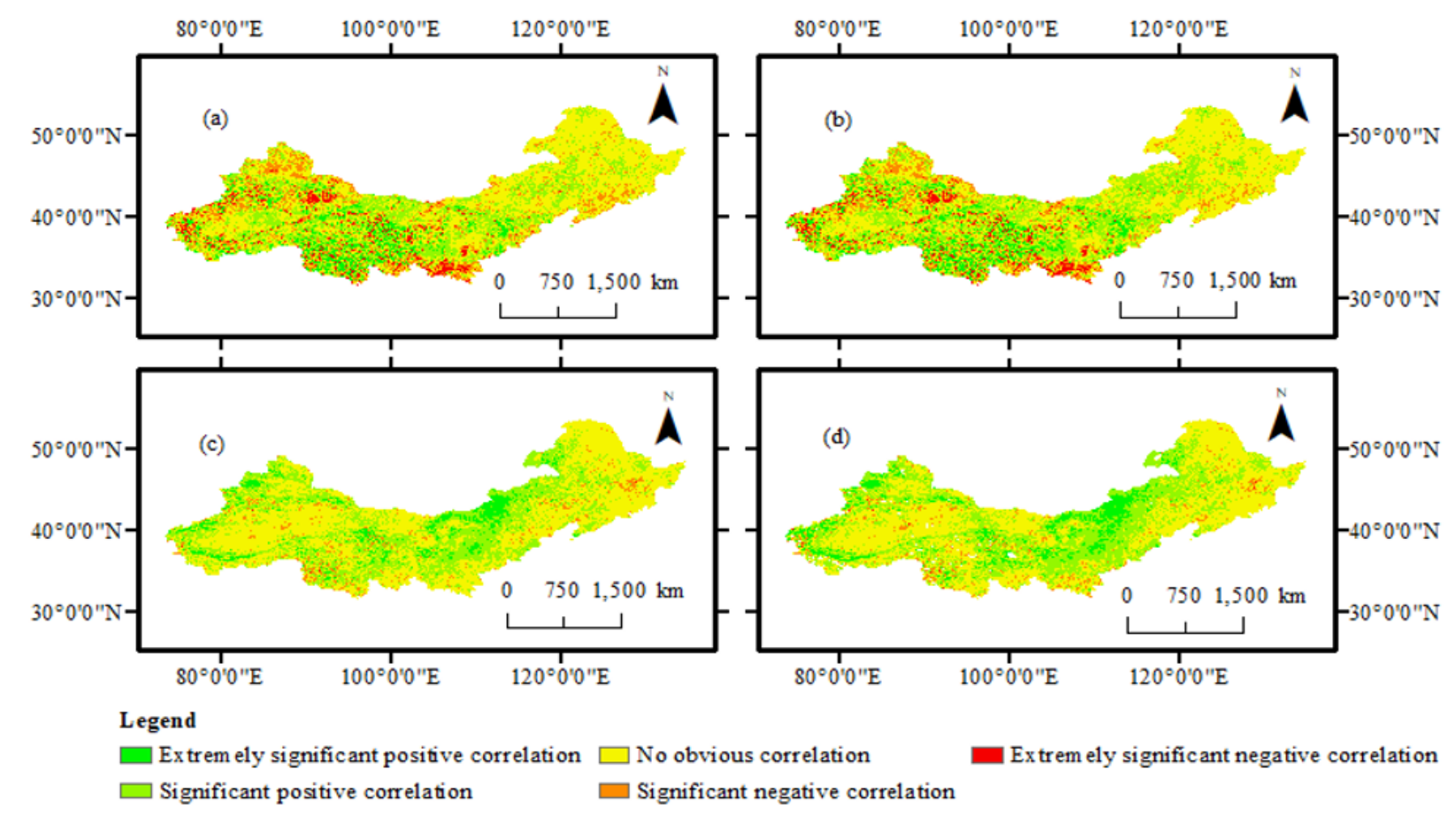

Fig.6. The correlation between NDVI and meteorological factors in the study area during the period of 1985-2015.( (a) and (b) are the correlation coefficient and partial correlation coefficient between NDVI and temperature, respectively; (c) and (d) are the correlation coefficient and partial correlation coefficient between NDVI and precipitation, respectively.)

The land cover types in the Tarim Basin are mainly desert and gobi, and the vegetation coverage is $<5 \%$. The areas with negative correlation (correlation $<-0.2$ ) between temperature and NDVI of northern vegetation are mainly distributed in junggar basin, turpan basin, loess plateau and Qinling mountains.

The spatial distribution of the NDVI and precipitation correlation coefficient (Fig.6c) and the partial correlation coefficient (Fig.6d) also have the same spatial distribution characteristics. The correlations and partial correlations between NDVI and precipitation showed no obvious significant correlations of $56.48 \%$ and $51.32 \%$, respectively (Table 2). It is mainly distributed in the Tarim Basin, the Turpan Basin and the western part of the Inner Mongolia Plateau. These areas are gobi or desert with sparse vegetation, which is not enough to promote the growth of vegetation within a limited range of rainfall. Heilongjiang, jilin and liaoning are the main crop areas.
Abundant rainfall will promote the growth of crops, but vegetation cover will not increase.

There is a significant positive correlation between precipitation and NDVI in northern grassland. The interannual variation of grassland vegetation growth is obviously dependent on the precipitation, such as the Junggar Basin, the Tarim Basin, the Tianshan Mountains and Inner Mongolia Grassland, the Qilian Mountains in the northeastern margin of the Qinghai-Tibet Plateau, and the Loess Plateau. There was a significant positive correlation (correlation and partial correlation) between NDVI and precipitation in these areas, accounting for $40.07 \%$ and $44.96 \%$ of the study area (Table 2). The area where precipitation is negatively correlated with the northern NDVI is small. It is mainly distributed in some scattered areas such as the Northeast Plain and Weinan. Overall, precipitation has a significant impact on the increase of NDVI in northern grassland.

Table 2. The area percentage of different correlation between NDVI and meteorological factors

\begin{tabular}{|c|c|c|c|c|}
\hline \multirow{2}{*}{ Correlation } & \multicolumn{2}{|c|}{ NDVI and Temperature } & \multicolumn{2}{c|}{ NDVI and Precipitation } \\
\cline { 2 - 5 } & $\begin{array}{c}\text { Correlation } \\
\text { analysis/\% }\end{array}$ & $\begin{array}{c}\text { Partial correlation } \\
\text { analysis/ } \%\end{array}$ & $\begin{array}{c}\text { Correlation } \\
\text { analysis/\% }\end{array}$ & $\begin{array}{c}\text { Partial correlation } \\
\text { analysis } / \%\end{array}$ \\
\hline Extremely significant positive correlation & 7.93 & 9.08 & 6.62 & 8.60 \\
\hline Significant positive correlation & 25.48 & 27.82 & 33.45 & 36.36 \\
\hline No obvious correlation & 49.04 & 46.79 & 56.48 & 51.32 \\
\hline Significant negative correlation & 13.05 & 11.98 & 3.39 & 3.65 \\
\hline Extremely significant negative correlation & 4.05 & 4.33 & 0.06 & 0.07 \\
\hline
\end{tabular}

\section{Conclusions and discussion}

This paper mainly studies the temporal and spatial trends of NDVI near 30 in northern China and the response to meteorological factors. In the past 30 years, the NDVI in 
the study area showed a slow-growth trend, but the fluctuations increased. The NDVI minimum appeared in 2004 at 0.4048 , the maximum at 0.4683 , and the NDVI growth rate at $1.5 \% \cdot(10 \mathrm{a})^{-1}$.

From the spatial distribution of NDVI trends, the area of vegetation improvement in the study area is much larger than the degraded area, accounting for $46.90 \%$ and $16.56 \%$, respectively, and the vegetation is effectively restored. The areas with no obvious changes are mainly deserts, Gobi and other sparsely populated areas, and short-term climate change has limited impact on the vegetation in the study area [9]. The areas with improved vegetation are mainly distributed in the Greater Khingan mountains area, Tai-hang Mountains, Inner Mongolia Plateau and Loess Plateau. These areas are located in alpine and highland areas, and the vegetation types are forests and grasslands. The increase of vegetation in the Loess Plateau may be the effect of ecological treatment projects [9]. The Tianshan Mountains, the Altay Mountains, and the Helan Mountains may be due to the complex terrain and the wide variety of vegetation and soils.

The average annual temperature in northern China increases year by year, while the precipitation increases slightly. As a typical region of arid, semi-arid and semihumid climate, the response of vegetation to climate change in northern China is different from other regions, and there are also some links [11]. Our research shows that precipitation and temperature in the northern region show different dominant positions in different terrains. The correlation and partial correlation between NDVI and temperature in higher alpine and subalpine regions are higher than that of precipitation (Fig.6). However, the correlation coefficient between NDVI and precipitation in northern grassland is higher than that of temperature, and the same for partial correlation analysis. It can be seen that the typical grassland and desert grassland in arid and semi-arid areas are very sensitive to the influence of precipitation, while the plateau grassland and woodland with higher altitudes are more sensitive to temperature.

\section{References}

1. H.D. Li, J. Jiang, B. Chen, Pattern of NDVI-based vegetation greening along an altitudinal gradient in the eastern Himalayas and its response to global warming. Environ monit assess, 188(3), 186 (2016)

2. Z.Q. Tan, H. Tao, J. Jiang, Influences of climate extremes on NDVI (Normalized Difference Vegetation Index) in the Poyang Lake Basin, China. Wetlands, 35,1033 (2015)

3. T. Higginbottom, E. Symeonakis, Assessing land degradation and desertification using vegetation index data: Current frameworks and future directions, Remote Sensing, 6(10), 9552-9575(2014)

4. Q. Guan, L. Yang, W. Guan, Assessing Vegetation Response to Climatic Variations and Human Activities: Spatiotemporal NDVI Variations in the Hexi Corridor and Surrounding areas from 2000 to
2010, Theoretical and Applied Climatology, 135(34), 1179-1193(2019)

5. X.W. Zhao, Q.Gao, Y.J. Yue, L. Duan, S.Pan, A System Analysis on Steppe Sustainability and Its Driving Forces-A Case Study in China. Sustainability, 10(1), 233(2018)

6. V. Muradyan, G. Tepanosyan, S. Asmaryan, Relationships between NDVI and climatic factors in mountain ecosystems: A case study of Armenia, Remote Sensing Applications: Society and Environment, 14, 158-169(2019)

7. M. C. Garcia-Aguirre, M. A. Ortiz, J.J. Zamorano, Vegetation and landform relationships at Ajusco volcano Mexico, using a geographic information system (GIS), Forest Ecology and management, 239(1-3), 1-12(2007)

8. C. Tucker, J. Pinzon, M. Brown, D. Slayback, E. Pak, R. Mahoney, E. Vermote, N. El Saleous, An extended AVHRR 8-km NDVI dataset compatible with MODIS and SPOT vegetation NDVI data. International Journal of Remote Sensing 26(20), 4485-4498(2005)

9. R.P. Zhang, Q.S.Feng, J.Guo, Spatio-temporal Changes of NDVI and Climatic Factors of Grassland in Northern China from 2000 to 2012, Journal of desert research, 5, 1403-1412(2015)

10. Q.X. Liu, R. D. Zhao, P. Shao, Temporal and spatial variation of vegetation coverage and its driving forces in the Loess Plateau from 2001 to 2015, Aird Land Geography, 41,101-110(2018)

11. M.G. Ma, V. Frank, Interannual variability of vegetation cover in the Chinese Heihe River Basin and its relation to meteorological parameters, Int J Remote Sens, 27, 3473-3486(2006) 